\title{
Flow-based titration with a colorimetric detection box using a smartphone for the determination of titratable acidity in coffee
}

\author{
Sarawut Somnam*, Miki Kanna \\ Department of Chemistry, Faculty of Science and Technology, Chiang Mai Rajabhat University, Chiang Mai \\ 50300 Thailand
}

*Corresponding author, e-mail: sarawut_som@cmru.ac.th

Received 18 Apr 2019

Accepted 22 Dec 2019

\begin{abstract}
Acidity is one of the key components for coffee quality which could be determined by titration. However, the endpoint observation with bare eyes might give error because of the dark color of coffee. Although this problem could be solved by using a $\mathrm{pH}$ meter, the calibration and maintenance processes including the cost of the device may be the drawbacks. In this research, a digital image colorimetric detection system was established with economic devices. The caption of image was carried out by a smartphone under the control of light intensity and focus range in a homemade detection box to assess the primitive color (red/green/blue or RGB) data from the color change of an acid-base indicator. In addition, to make process more comfortable and reduce the detection time, the flow-based technique was also applied to the setup for the signal monitoring process. The method was highly reproducible with \%RSD less than $1(n=11)$ and detection time of $24 \mathrm{~s}$ was achieved. From the analyses of roasted coffee beans, the results obtained agreed well with those from the potentiometric standard method.
\end{abstract}

KEYWORDS: titratable acidity, flow-based titration, smartphone, coffee

\section{INTRODUCTION}

Coffee acidity typically associated with the perceived flavors and aroma, including directly attributed to acids found in the coffee beans. Acidity can enliven the attractive taste of coffee as bright, tart and lush. However, acidity in coffee does not mean to a sour sensation, even though types of acids can contribute to the sourness [1,2]. Chlorogenic acids are a group of phenolic compounds abundantly found in coffee [3]. Nevertheless, the roasting process could be the cause of degradation of chlorogenic acid to form quinic acid, caffeic acid, lactones, and other phenol derivatives through Millard and Strecker's reaction, which result in increased bitterness, astringency, aroma, and color [4-6]. Several organic acids present in coffee, such as citric, malic, acetic, oxalic, succinic and tartaric acid, with the balance proportion, provide further layers of complexity and depth in the cup. Therefore, the determination of acidity is important as one of the key parameters that influence the coffee quality $[7,8]$.

Titration using the standardized $\mathrm{NaOH}$ as the titrant is the standard method for the determination of acidity in beverages [9]. However, the observa- tion of the endpoint via bare eyes might be biased when applying to dark-colored samples, such as coffee. Even the potentiometric titration using a pH meter can get around a problem, the sophisticated procedures both the calibration of a $\mathrm{pH}$ electrode and keeping the electrode in an electrolyte after use, including the cost of the $\mathrm{pH}$ meter should be recognized. Apart from a titration technique, there are some reported techniques, such as near infrared spectroscopy (NIR) [10], ion chromatography (IC) [11], and high performance liquid chromatography (HPLC) [12-15], which are also effectively applied to distinguish and quantitate the acids in coffee. However, the expensive instrument and time-consuming procedures should be the obstacle for the laboratory that lacks budgets and skilled labor.

With the reasons of accuracy, expense, and convenience, in this work, a portable multi-function daily usage device with high performance of the camera, i.e., a smartphone, is employed as a detector to monitor the RGB (red, green, blue) primitive color of indicator when mix to the sample during titration based on a digital image based method. The digital image which is a pixel (the smallest color 
unit) could be captured via the digital devices by mixing of RGB color in each pixel which is utilized in displays or computer graphic applications [16]. Each color is defined in different intensities with values from sensor reading vary from 0-255 (8 bit). Hence, the total number from the primary color combination is $2^{8} \times 2^{8} \times 2^{8}=16788216$ colors, of which close to the visual ability of human eyes [17, 18]. The combination of maximum values of $R, G$, and $\mathrm{B}$ gives white color, while zero intensity for each component gives black.

The image of the coffee sample mixed with an indicator is continuously captured at each of the fixed volumes of titrant added. RGB values of images are assessed via the costless downloadable application and use to plot the titration curve. In order to make more precise and comfort, the experiment was carried out under controlling of light intensity and focus range with a home-made RGBdetection box, which is compact, portable, robust, and can support the different sizes of a smartphone. The flow-based technique is also applied for aspirating/propelling the sample solution into a sample cell inside the detection box via a tiny PTFE tube (instead of taking an aliquot of solution by a glassware) that could avoid the spill of chemicals and also reduce the detection time and chemical quantity. Therefore, the digital imagebased titration setup by applying the flow system to transfer the solution for the detection step has been developed for the determination of acidity in coffee. The system is assembled with a low-cost device but easy to use, no need for the calibration and maintenance procedures, and also gain high accuracy of endpoint observation based on the primary color even applying to the dark-colored sample.

\section{MATERIALS AND METHODS}

\section{Chemicals}

All solutions were freshly prepared with de-ionized water and analytical grade chemicals.

Primary standard solutions of $0.09980 \mathrm{M}$ $\mathrm{KHC}_{8} \mathrm{H}_{4} \mathrm{O}_{4}$ or $\mathrm{KHP}$ (Rankem) were prepared for the standardization procedure. About $0.1 \mathrm{M} \mathrm{NaOH}$ (RCI Labscan) and $0.1 \mathrm{M} \mathrm{HCl} \mathrm{(RCI} \mathrm{Labscan)} \mathrm{were}$ standardized before used as the standard reagent and titrant, respectively, for the back titration of acidity in coffee. About $0.1 \% \mathrm{w} / \mathrm{v}$ of bromothymol blue (Ajax Finechem), methyl red (RCI Labscan) and phenolphthalein (RCI Labscan) were used as indicators in the titration.
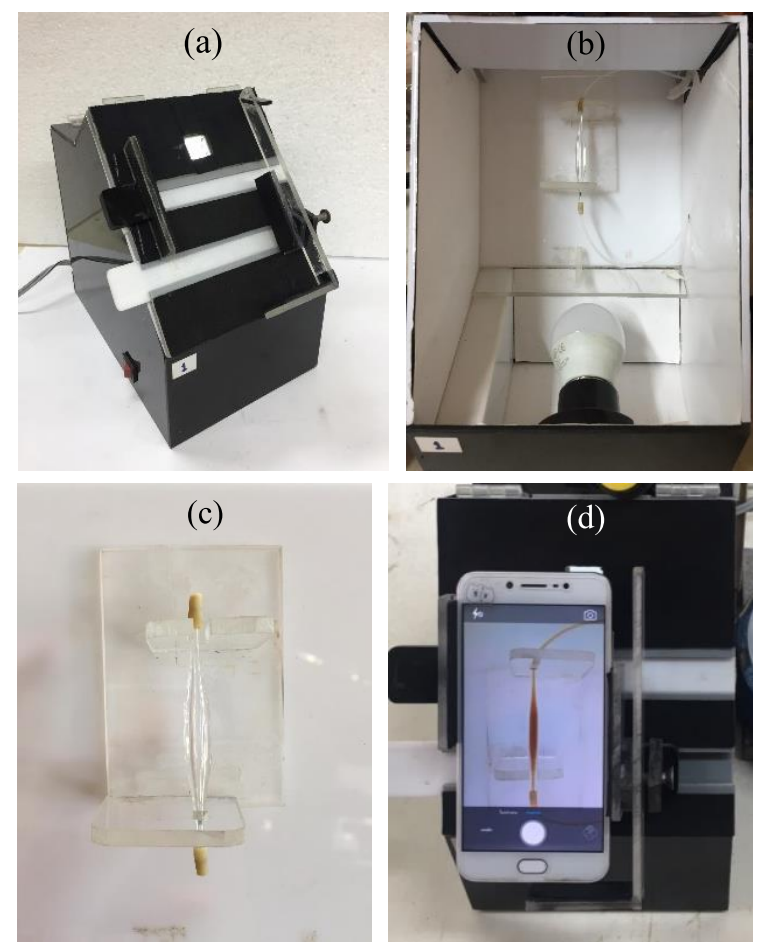

Fig. 1 (a) An acrylic RBG-detector box, (b) the inside of the box, (c) acrylic sample holder with a flow cell, (d) the phone placed on the box.

\section{Sample preparation}

Twelve Arabica coffee samples, consisted of light/medium/dark roasted beans, from three cultivated areas in the north of Thailand were used in this study. All seeds were ground in a coffee grinder to pass a $0.5 \mathrm{~mm}$ sieve. Ground coffee of $20 \mathrm{~g}$ was boiled with $500 \mathrm{ml}$ water for $10 \mathrm{~min}$. The solution was then filtered through a filter paper no. 1 and left at room temperature before use.

\section{RGB-detection box}

A home-made RGB-detection box was built with the aims of preventing the interfering of ambient light while capturing a digital image with a mobile phone for color intensities measurement. The detection box was designed for comfortable, compact, robust, applicable to flow-based technique, and could support the various sizes of the mobile phone [19]. The black opaque acrylic sheet (thickness 3.0$4.0 \mathrm{~mm}$ ) was used for assembling the whole structure $(14.0 \mathrm{~cm} \times 22.0 \mathrm{~cm} \times 21.0 \mathrm{~cm})$ as illustrated in Fig. 1a. The inside of the box was covered with a white paper to provide uniform illumination and attached 3 W LED light bulb (Lumax) at the bottom 
as shown in Fig. 1b. The box was drilled for two small size holes at the right side to insert PTFE tubing (i.d. $0.795 \mathrm{~mm}$ ) for flow inlet and outlet. A home-made glass flow cell with an inner volume of $220 \mu 1,5.0 \mathrm{~mm}$ i.d., and length of $70 \mathrm{~mm}$ as shown in Fig. 1c was fitted on the acrylic holder which also attached to the acrylic sheet $(14.0 \mathrm{~cm} \times 14.5 \mathrm{~cm})$, which was easily to put in and out via a hinged cover lid on the top of the box. A foldable front cover lid opposite to the part of sample holder, was drilled $(1.5 \mathrm{~cm} \times 1.5 \mathrm{~cm})$ to place the Android smartphone (Vivo) to capture an image. A mobile phone holder was also made from an acrylic sheet which was moveable from left-right and upward-downward by placing the lens fit onto the detection hole (Fig. 1d) with a fixed distance between the flow cell and lens at $80 \mathrm{~mm}$. The moveable phone holder provided a maximum area of $630 \mathrm{~cm}^{2}$ which was supportable to the various sizes of the phone. Rubber sheet was glued on the front lid that contacts to phone to protect from scratch.

\section{Acquisition of RGB values}

The RGB values were assessed by a costless downloadable application, i.e., Rgb Color Picker, which could be downloaded from Play Store application. After capturing the image, the application could display the color value with the most common color models, i.e., RGB, CMYK, HSL, HSV, and YUV, and even gave a name to a color. For the attainment of RGB values in this work with the mentioned application, after the solution flowed fully into a flow cell, the color capture was done in RGB mode. Each of R, G, and B defined the intensity of color as the integer from 0-255.

\section{Flow-based titration setup for the determination of titratable acidity in coffee}

Since the low content of acidity is found in coffee, in this work, back titration technique was employed by adding the excess amount of $\mathrm{NaOH}(25 \mathrm{ml}$, $0.09910 \mathrm{M}$ ) into a $25 \mathrm{ml}$ of brewed coffee solution in a $250 \mathrm{ml}$ beaker. An aliquot of $50 \mathrm{ml}$ deionized water and three drops of indicator were added and then titrated to the standardized $0.0995 \mathrm{M} \mathrm{HCl}$. The color change of indicator was detected for RGB values when every portion of titrant $(1.00 \mathrm{ml})$ was added into the sample until reached $50 \mathrm{ml}$. The flow-based titration setup was assembled as illustrated in Fig. 2.

A dual channel peristaltic pump (Ismatec) was employed to simultaneously aspirate the air and the solution with a constant flow rate at $1.35 \mathrm{ml} / \mathrm{min}$.

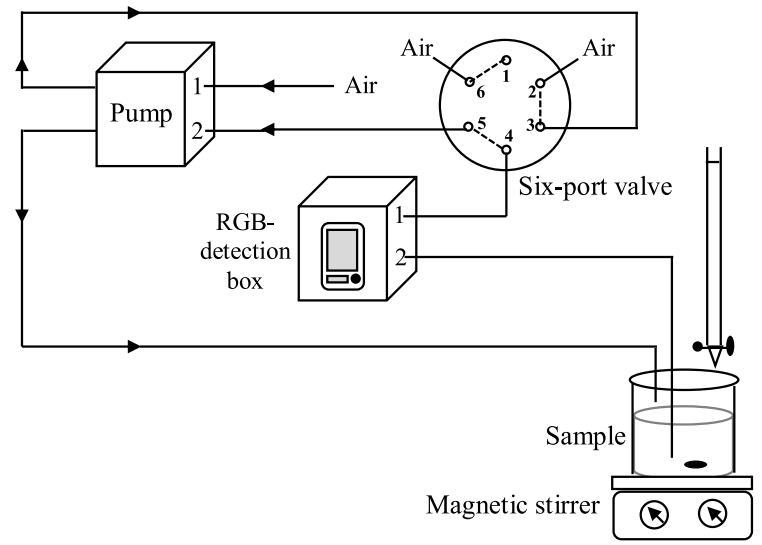

Fig. 2 Schematic diagram of a flow-based titration setup with RGB-detection box.

Six-port valve (Upchurch Scientific) was used for controlling the flow direction. The operation sequence is briefly described as follows. After a portion of a defined titrant volume was filled into the mixture solution, between the coffee sample, $\mathrm{NaOH}$, and indicator, in a beaker which was continuously stirred, a mixed solution was then drawn up by a peristaltic pump though a PTFE tubing. Solution was aspirated through a flow cell in the detection box for monitoring RGB signals, a valve at positions 4 and 5, respectively, before flowed back to a beaker. After obtaining that RGB values, switched the valve to push the air through positions 3-4 and 5-6 to propel the whole solution in the tubing back into a beaker for the next cycle detection. The flow-based titration had a detection frequency of 24 s/cycle.

The titration curves were constructed by plotting the titrant volume versus RGB values to gain the equivalent point from the inflection point. All results were also compared with those of potentiometric titration method by monitoring with a benchtop pH meter (Consort).

\section{RESULTS AND DISCUSSION}

\section{Effect of ambient light on RGB-detection box}

To ensure that the ambient light could not percolate through a detection box while monitoring, different light condition, i.e., bright room (turn on the light), dim room (turn off the light), and outdoor (at noon) were investigated by analyzing the color solution of $0.02 \% \mathrm{w} / \mathrm{v}$ bromothymol blue buffered pH 5 and 9. It is well known that bromothymol blue has the color transformation from yellow in acidic solution (according to the mixture between the primitive color of red (R) and green (G)) to blue 
Table 1 Effect of ambient light on the monitoring of color intensities by RGB-detection box.

\begin{tabular}{|c|c|c|c|c|c|c|}
\hline \multirow{3}{*}{$\begin{array}{l}\text { Light } \\
\text { condition }\end{array}$} & \multicolumn{6}{|c|}{ Average intensity of color (mean \pm SD, RSD) } \\
\hline & \multicolumn{3}{|c|}{ pH 5.0} & \multicolumn{3}{|c|}{ pH 9.0} \\
\hline & $\mathrm{R}$ & G & B & $\mathrm{R}$ & G & B \\
\hline Bright & $151.4 \pm 1.1,0.7 \%$ & $69.6 \pm 0.7,1.0 \%$ & $12.6 \pm 0.5,4.0 \%$ & $25.4 \pm 1.1,4.4 \%$ & $89.8 \pm 0.6,0.7 \%$ & $134.2 \pm 0.8,0.6 \%$ \\
\hline Dim room & $147.5 \pm 0.9,0.6 \%$ & $64.8 \pm 0.6,0.9 \%$ & $10.0 \pm 0.4,4.5 \%$ & $20.1 \pm 0.7,3.5 \%$ & $80.6 \pm 0.8,1.0 \%$ & $138.2 \pm 0.9,0.6 \%$ \\
\hline Outdoor & $154.1 \pm 1.0,0.6 \%$ & $68.7 \pm 0.9,1.3 \%$ & $13.5 \pm 0.5,3.7 \%$ & $25.8 \pm 0.6,2.3 \%$ & $90.8 \pm 1.1,1.2 \%$ & $139.9 \pm 0.7,0.5 \%$ \\
\hline
\end{tabular}

in basic solution (according to blue (B) primitive color) that could indicate the effect of ambient light to the monitor of all primitive color by the detection box. The average RGB values of eleven replicates at different ambient light condition are summarized in Table 1. The result of high precision ( RSD < 5\%) indicates that the ambient light could not intrude the detection box while the LED light controlled constant illumination inside.

\section{The suitable indicator for the acidity determination in coffee}

Although phenolphthalein was used as the indicator in the standard method for the acidity determination, however, the observation of the change of color in a dark colored sample with RGB detection using a smartphone might cause an error. Three indicators, i.e., methyl red, bromothymol blue, and phenolphthalein, were chosen to study the suitable indicator that is appropriate to the strong acid-strong base titration and had the difference in both $\mathrm{pH}$ transition range and color change as the theoretical aspect; 4.4-6.2 (red to yellow), 6.0-7.6 (yellow to blue), and 8.0-10.0 (colorless to pink), respectively. The dark roasted coffee sample that provided the darkest colored solution was also used in this experiment by the aforementioned titration procedure. Because this study was the back titration technique by first mixing between excess $\mathrm{NaOH}$ and coffee sample before titrated with $\mathrm{HCl}$, the colors of indicator both in basic and acidic solution at before and after equivalence point were obtained, respectively. However, we found similar color change of all indicators in titration curves as depicted in Fig. 3.

Before the equivalence point, the mixed color of coffee and an indicator in a basic solution provided the dark color that affected to the low RGB values (R/G/B values of white and black were $255 / 255 / 255$ and 0/0/0, respectively). However, R value always presented the highest due to the darkbrown color of coffee. When surpassed the equivalence point, the coffee color turned to lighter brown resulted from the dilution by addition of the titrant. But because the coffee color was still darker than the
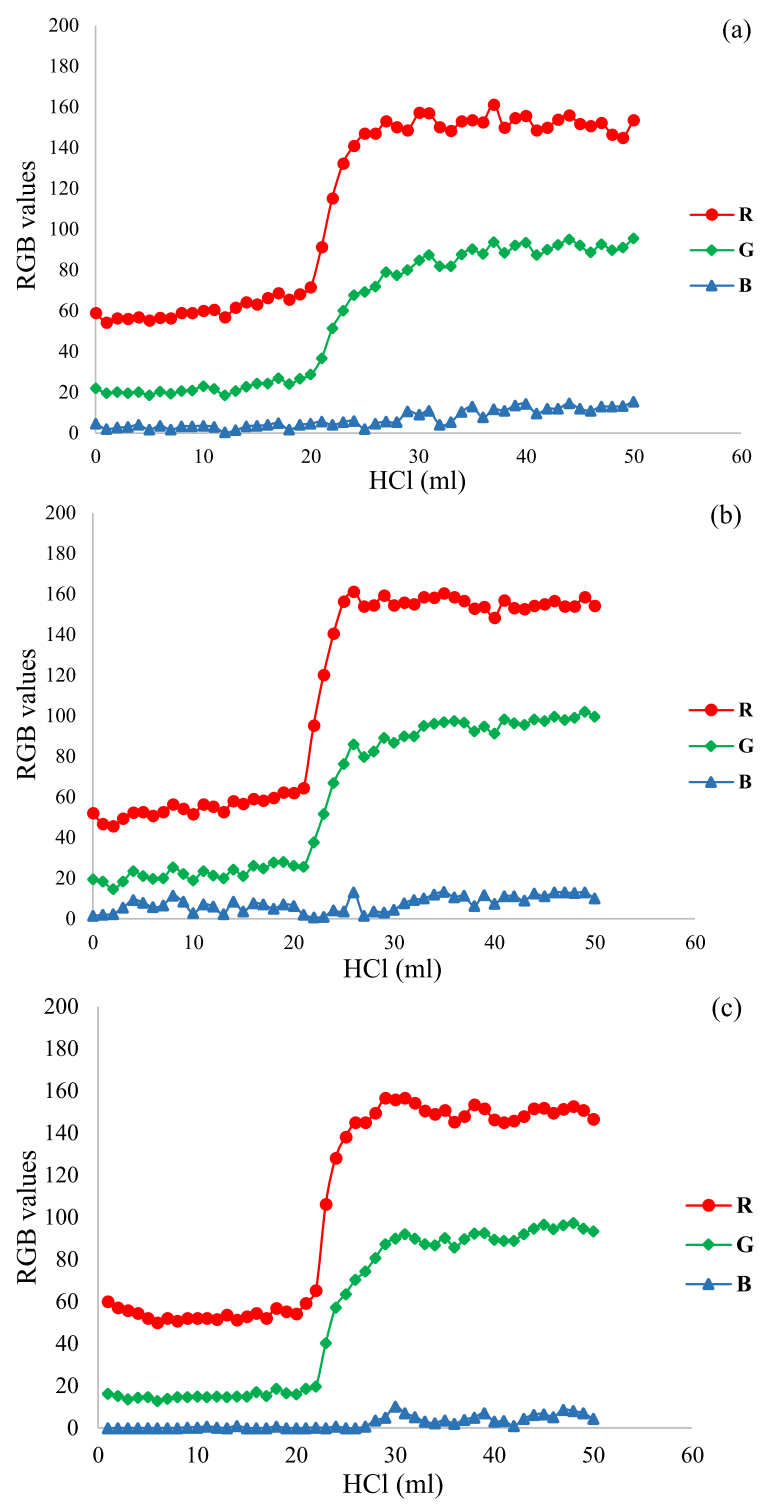

Fig. 3 Titration curve from using the indicator with the appearance of coffee color (a) methyl red, (b) bromothymol blue, and (c) phenolphthalein.

indicator color, with methyl red and bromothymol blue turned to red and yellow, respectively, such occurrence led to the abrupt increase in $\mathrm{R}$ and $\mathrm{G}$ 

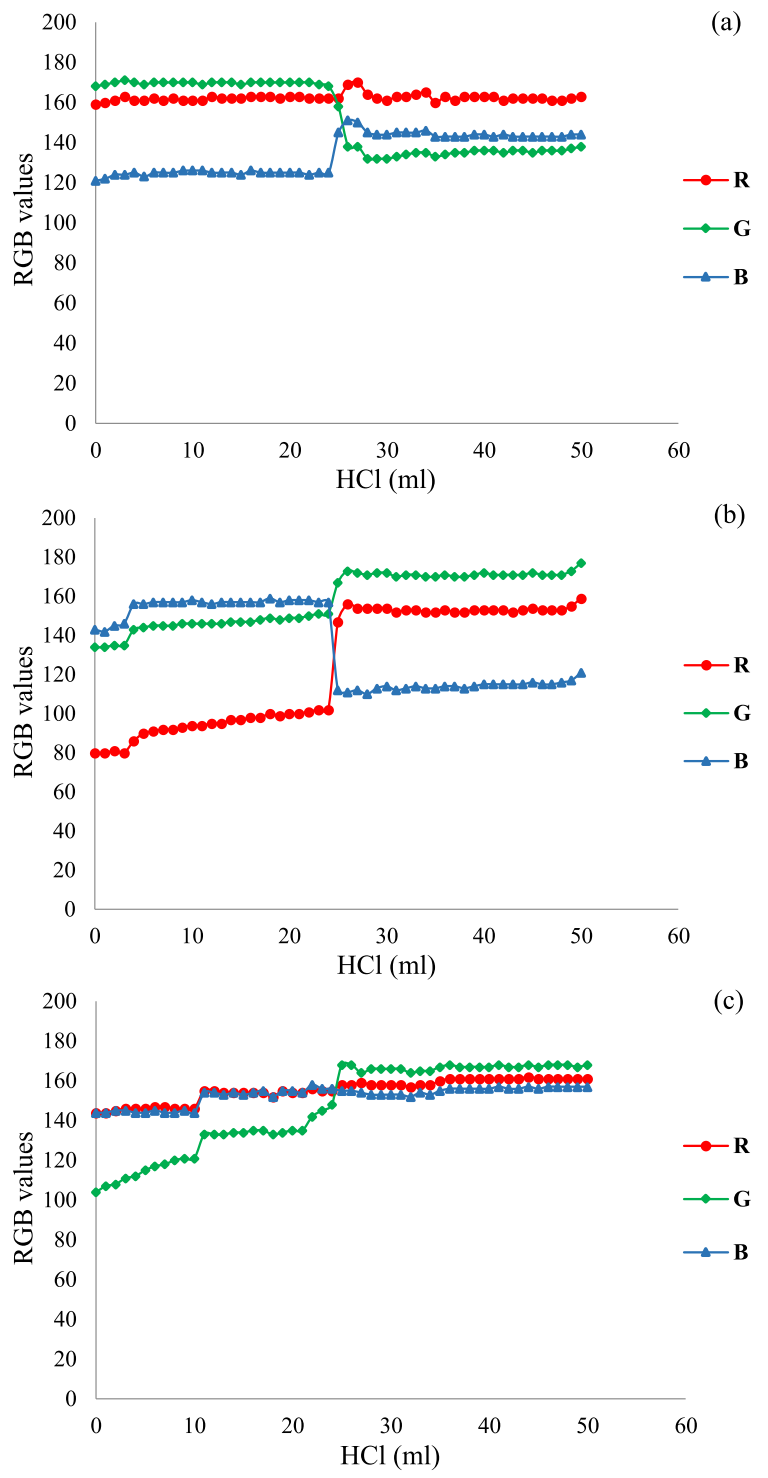

Fig. 4 Titration curve from using the indicator without the coffee color (a) methyl red, (b) bromothymol blue, and (c) phenolphthalein.

values while $B$ value was constant throughout the signal detection process. For phenolphthalein which turned to colorless, the solution exhibited only the light-brown coffee color. RGB values, then, changed according to the previously mentioned indicators.

Therefore, to ensure that the coffee color had more effect on the change of the titration curve, blank titration to study the color change of each indicator was also done without the addition of coffee. The titration curves are illustrated in Fig. 4 that showed the distinct characters for each of the indicators when comparing to the appearance of coffee color.

Fig. 4a showed that $R$ and $G$ values were higher than $B$ value before equivalence point due to the yellow color of the indicator in basic solution. It could be described that the mixture between the additive color of $\mathrm{R}$ and $\mathrm{G}$ provided the yellow in accordance with the color of methyl red at the $\mathrm{pH}$ higher than 6.2. Then after equivalence point, the $G$ value decreased due to the color transformation to red. For the change of RGB values of bromothymol blue as shown in Fig. 4b, before equivalence point with the blue color basic solution, B and G values were similarly high while $\mathrm{R}$ value was much lower. When $\mathrm{pH}$ is below 6 , the color turns to yellow, $\mathrm{R}$ value then obviously increased while $B$ value dropped in the same way of the change in methyl red. In the case of phenolphthalein (Fig. 4c), because of the pink color in basic solution before the equivalence point, $R$ and $B$ values were constant while $G$ value decreased. After the color turns to colorless in acidic solution, then the captured color was the white color of the covered paper in the detection box and affected to the similar values of RGB [20].

With the influence of coffee color, even if different types of indicators were used, the change in the shapes of titration curves was somewhat identical with obvious inflection at the equivalence point. This indicated that all the studied indicators could be applied to the proposed analytical method. However, phenolphthalein was chosen as the appropriate indicator for further experiment because it was a normally used indicator in the standard method and also it turned to colorless at the equivalence point which did not interfere with the coffee color. Only $\mathrm{R}$ primitive color was also used to plot the titration curve in the next study because it showed the most distinct change.

\section{Effect of $\mathrm{NaOH}$ concentration}

With the use of back titration technique and low amount of acidity in the coffee sample, the suitable concentration of $\mathrm{NaOH}$ should be investigated by varying its concentration. Titration curves were illustrated in Fig. 5.

The effect of $\mathrm{NaOH}$ concentrations was shown in Fig. 5 by the different shape of titration curves. The higher concentration which had the higher number of moles of the titrant could provide the larger changes on signal around the equivalence point for every type of titration. As the concentration increased, the sharpness of the inflection point also increased. Therefore, $0.1 \mathrm{M} \mathrm{NaOH}$ was selected with the use of the same concentration of $\mathrm{HCl}$. 


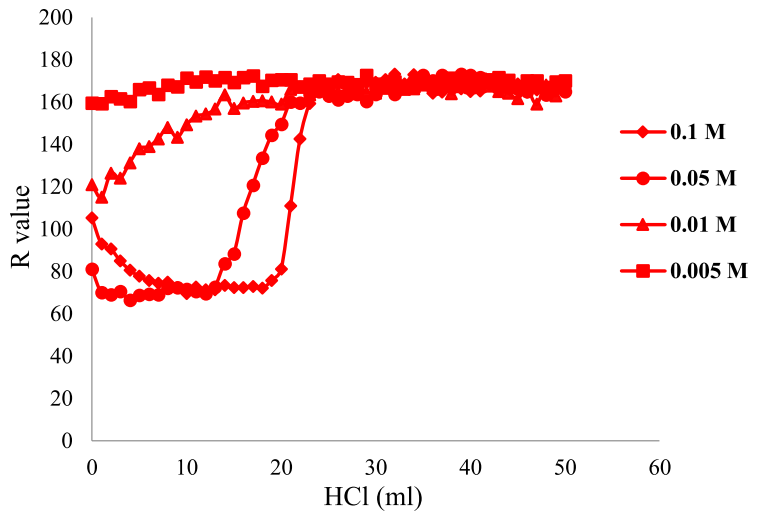

Fig. 5 Titration curves obtained from varying the concentration of $\mathrm{NaOH}$ when titrated with $\mathrm{HCl}$.

Table 2 The amount of titratable acidity in coffee using the flow-based titration setup and the potentiometric titration.

\begin{tabular}{llc}
\hline \multirow{2}{*}{$\begin{array}{l}\text { Roasted coffee } \\
\text { sample }\end{array}$} & \multicolumn{2}{c}{ Titratable acidity (\% w/v) } \\
\cline { 2 - 3 } & Flow-based & Potentiometric \\
\hline Mae Salong & & \\
Light roast & $0.77 \pm 0.03$ & $0.78 \pm 0.03$ \\
Medium roast & $0.67 \pm 0.02$ & $0.67 \pm 0.01$ \\
Dark roast & $0.55 \pm 0.01$ & $0.54 \pm 0.01$ \\
\hline Inthanon & & \\
Light roast & $0.63 \pm 0.02$ & $0.63 \pm 0.01$ \\
Medium roast & $0.57 \pm 0.02$ & $0.58 \pm 0.02$ \\
Dark roast & $0.54 \pm 0.01$ & $0.52 \pm 0.01$ \\
\hline Chang Kian & & \\
Light roast & $0.70 \pm 0.04$ & $0.71 \pm 0.03$ \\
Medium roast & $0.62 \pm 0.02$ & $0.61 \pm 0.01$ \\
Dark roast & $0.50 \pm 0.01$ & $0.52 \pm 0.01$ \\
\hline
\end{tabular}

\section{Determination of titratable acidity in coffee}

RGB-detection box for a flow-based titration setup under the above motioned selected conditions was applied for sample analysis by comparing the analytical result with the potentiometric titration. The volumes at equivalence point from titration curve were calculated in order to assay the contents of acidity in coffee as summarized in Table 2. The analytical results from RGB detection using a smartphone were statistically compared by paired $t$-test at $95 \%$ confidence level to the assay results of a $\mathrm{pH}$ meter, no significant difference between the two methods was found. Therefore, it might be estimated that the proposed setup could be effectively employed to the determination of acidity for the dark-colored sample.

\section{CONCLUSION}

The developed RGB detection using a smartphone to monitor RGB primitive color for the flow-based titration was achieved for the determination of acidity in dark-colored samples, such as coffee. RGB value could be successfully applied to build a titration curve which gave the agreeable results when compared to the results obtained from a $\mathrm{pH}$ meter. The suitable indicator and $\mathrm{NaOH}$ concentration were investigated in order to increase analytical effectiveness. The proposed analyzer showed considerable advantages, e.g., comfort, portable, robust, and cheap analysis.

Acknowledgements: Research funding from the National Research Council of Thailand (NRCT) is gratefully acknowledged. We also thank the Center of Science and Technology, Chiang Mai Rajabhat University for equipment support.

\section{REFERENCES}

1. Jagan Mohan Rao L, Ramalakshmi K (2011) Introduction to coffee. In: Jagan Mohan Rao L, Ramalakshmi K Recent (eds) Recent Trends in Soft Beverages, Woodhead Publishing India, India, pp 3-25.

2. Cheng B, Furtado A, Smyth HE, Henry RJ (2016) Influence of genotype and environment on coffee quality. Trends Food Sci Tech 57, 20-30.

3. Ribeiro VS, Leitão AE, Ramalho JC, Lidon FC (2014) Chemical characterization and antioxidant properties of a new coffee blend with cocoa, coffee silverskin and green coffee minimally processed. Food Res Int 61, 39-47.

4. Duarte GS, Pereira AA, Farah A (2010) Chlorogenic acids and other relevant compounds in Brazilian coffees processed by semi-dry and wet post-harvesting methods. Food Chem 118, 851-855.

5. Stefanello N, Spanevello RM, Passamonti S, Porciúnculad L, Bonan CD, Olabiyi AA, da Rocha JBT, Assmann CE, et al (2019) Coffee, caffeine, chlorogenic acid, and the purinergic system. Food Chem Toxicol 123, 298-313.

6. de Melo Pereira GV, de Carvalho Neto DP, Magalhäes Júnior AI, Vásquez ZS, Medeiros ABP, Vandenberghe LPS, Soccol CR (2019) Exploring the impacts of postharvest processing on the aroma formation of coffee beans - A review. Food Chem 272, 441-452.

7. Gloess AN, Schönbächler B, Klopprogge B, D'Ambrosio L, Chatelain K, Bongartz A, Strittmatter A, Rast M, et al (2013) Comparison of nine common coffee extraction methods: instrumental and sensory analysis. Eur Food Res Technol 236, 607-627.

8. Rodrigues CI, Marta L, Maia R, Miranda M, Ribeirinho M, Máguas C (2007) Application of solid-phase extraction to brewed coffee caffeine and organic acid 
determination by UV/HPLC. J Food Compos Anal 20 , 440-448.

9. AOAC (1990) Coffee and tea. In: Dick RH (ed) Official Method of Analysis of the Association of Official Analytical Chemists, 15th edn, VA, USA, pp 757-758.

10. Santos JR, Lopo M, Rangel AOSS, Lopes JA (2016) Exploiting near infrared spectroscopy as an analytical tool for on-line monitoring of acidity during coffee roasting. Food Control 60, 408-415.

11. Gloess AN, Vietri A, Wieland F, Smrke S, Schönbächler B, López JS, Petrozzi S, Bongers S, et al (2014) Evidence of different flavour formation dynamics by roasting coffee from different origins: On-line analysis with PTR-ToF-MS. Int J Mass Spectrom, 3656, 324-337.

12. Evangelista SR, Silva CF, da Cruz Miguel MGP, de Souza Cordeiro C, Pinheiro ACM, Duarte WF, Schwan RF (2014) Improvement of coffee beverage quality by using selected yeasts strains during the fermentation in dry process. Food Res Int 61, 183-195.

13. Smrke S, Kroslakova I, Gloess AN, Yeretzian C (2015) Differentiation of degrees of ripeness of Catuai and Tipica green coffee by chromatographical and statistical techniques. Food Chem 174, 637-642.

14. Bressani APP, Martinez SJ, Evangelista SR, Dias DR, Schwan RF (2018) Characteristics of fermented cof- fee inoculated with yeast starter cultures using different inoculation methods. LWT Food Sci Technol 92, 212-219.

15. Craig AP, Fields C, Liang N, Kitts D, Erickson A (2016) Performance review of a fast HPLC-UV method for the quantification of chlorogenic acids in green coffee bean extracts. Talanta 154, 481-485.

16. Makled WA, Tahoun SS (2015) Digital quantification of the miospore coloration to assess the thermal maturity: Novel RGB-based measuring technique. Mar Petrol Geol 67, 1-15.

17. Lima MB, Andrade SIE, Barreto IS, Almeida LF, Araújo MCU (2013) A digital image-based microflow-batch analyser. Microchem $J$ 106, 238-243.

18. Andrade SIE, Lima MB, Barreto IS, Lyra WS, Almeida LF, Araújo MCU, Silva EC (2013) A digital imagebased flow-batch analyzer for determining $\mathrm{Al}(\mathrm{III})$ and $\mathrm{Cr}(\mathrm{VI})$ in water. Microchem $J$ 109, 106-111.

19. Somnam S, Kanna M, Jakmunee J (2019) Application of smartphone to increase effectiveness in the determination of soil $\mathrm{pH}$ by using indictors. Chiang Mai J Sci 46, 733-740.

20. Chanla J, Kanna M, Jakmunee J, Somnam S (2019) Application of smartphone as a digital image colorimetric detection for batch and flow-based acid-base titration. Chiang Mai J Sci 46, 975-986. 